\title{
Immunotherapies for Anti-NMDA Receptor Encephalitis: Multicenter Retrospective Pediatric Cohort Study in China
}

\section{Shiqi Guang}

Xiangya Hospital Central South University

Jiannan Ma

Chongqing Medical University Affiliated Children's Hospital

\section{Xiaotun Ren}

Beijing Children's Hospital

Shuizhen Zhou

Children's Hospital of Fudan University

Jian Yang

Capital Institute of Pediatrics

Jianzhao Zhang

Capital Institute of Pediatrics

Xiaoshuang Cao

Xiangya Hospital Central South University

Linxiu Zhong

Xiangya Hospital Central South University

Xiao Ding

Chongqing Medical University Affiliated Children's Hospital

Xiaosu Wang

Chongqing Medical University Affiliated Children's Hospital

Changhong Ren

Beijing Children's Hospital

Weihua Zhang

Beijing Children's Hospital

Linmei Zhang

Children's Hospital of Fudan University

Min Zhang

Children's Hospital of Fudan University

Jing Sun

Capital Institute of Pediatrics

Miriam Kessi 
Xiangya Hospital Central South University

\section{Fei Yin}

Xiangya Hospital Central South University

Jing Peng ( $\sim$ pengjing627@126.com )

Xiangya Hospital Central South University https://orcid.org/0000-0002-7752-6962

\section{Yuwu Jiang}

Peking University First Hospital

\section{Research}

Keywords: Anti-NMDAR receptor encephalitis, immunotherapy, autoimmune encephalitis, pediatrics

Posted Date: March 5th, 2021

DOl: https://doi.org/10.21203/rs.3.rs-276564/v1

License: (c) (i) This work is licensed under a Creative Commons Attribution 4.0 International License. Read Full License 


\section{Abstract}

\section{Background}

Anti-N-methyl-D-aspartate receptor (NMDAR) encephalitis has been discovered and termed more than a decade, but the establishment of standardized immunotherapy protocol for pediatric patients still needs more clinical evidence. To help move this forward, we investigated the current status of immunotherapies for pediatric anti-NMDAR encephalitis in 6 tertiary medical centers across China and evaluated how different immunotherapy strategies affected patient outcomes.

Methods

A multicenter, retrospective study was conducted on pediatric patients diagnosed with anti-NMDAR encephalitis between November 2011 and December 2018. The clinical records including clinical manifestations, immunotherapy strategies, and outcomes were collected and analyzed. Treatment response and outcome were evaluated using mRS. Outcomes among the treatment groups were analyzed with the Chi-squared test or Fisher's exact test. $p<0.05$ was considered significant.

Results

A total of 386 patients were included in our study and the median onset age was 7.89 (range 0.5-18) years. All patients received first-line immunotherapy and the majority $(341,88.3 \%)$ used the standard combination of methylprednisolone pulses and intravenous immunoglobulins, but 211 patients did not show satisfactory improvement ( $\mathrm{mRS} \geq 3$ ). Mainly three treatment strategies were applied after first-line immunotherapy: second-line immunotherapy, repetitive first-line immunotherapy, and maintaining oral prednisolone. For patients with $\mathrm{mRS} \geq 4$ after first-line immunotherapy, the incidence of poor outcome $(m R S \geq 3)$ in oral prednisolone group was higher than that in other treatment groups $(0.025<p<0.05)$. No difference in complete recovery rate $(\mathrm{mRS}=0)$ was found between patients receiving second-line and repetitive first-line immunotherapy, or patients using long-term and short-term prednisolone. The relapse rate of oral prednisolone group was higher than that of other treatment groups $(p<0.01)$, but the relapse rate of patients using long-term and short-term prednisolone had no statistical difference.

Conclusions

For patients with $m R S \geq 4$ after first-line immunotherapy, second-line immunotherapy is recommended. When second-line immunotherapy is not applicable, repetitive first-line immunotherapy can be considered as an option. Both second-line and repetitive first-line immunotherapy are beneficial to reduce relapse rate. The duration of sequential oral prednisolone can be shortened after fully evaluating patients' conditions.

\section{Background}


Anti-N-methyl-D-aspartate receptor (NMDAR) encephalitis was first termed in 2007 due to the discovery of specific autoantibodies to NMDA receptors in a series of patients who developed a constellation of neuropsychiatric symptoms, associated with ovarian teratoma $(1,2)$.

A large series study of 577 patients revealed approximately $80 \%$ of patients improved or recovered after immunotherapies and tumor removal if it was applicable (3).

Compared to adult patients, pediatric patients showed a different clinical symptom profile and lower tumor association rate, and they may have different response to immunotherapy and better outcome (4). Although the frame of immunotherapy is well defined, in which, patients receive first-line immunotherapy and then proceed to second-line immunotherapy if clinical improvement is not satisfactory, the evidence of effectiveness and superiority of any treatment regimen is lacking. Currently, the most widely accepted first-line immunotherapy is the high-dose intravenous methylprednisolone pulses (MEP) alone or combined with intravenous immunoglobulins (IVIG); rituximab for second-line immunotherapy is another area of agreement $(5,6)$. However, our survey on treatment strategies of pediatric neurologists in China revealed, a considerable proportion of clinical practitioners would repeat first-line immunotherapy once before considering second-line immunotherapy (7). The survey of Bartolini et al. also showed pediatric neurologists were more likely to repeat first-line immunotherapy compared to adult neurologists and pediatric rheumatologists (5).

Furthermore, high dose of oral prednisolone followed by tapering is another major maintenance treatment after first-line immunotherapy, but the dosage and duration are also open to discussion. How these clinical decisions will affect the outcome of patients is still a pending question. Consequently, we performed this nationwide, multicenter study with 386 pediatric patients involved, aiming to investigate the current immunotherapy strategies for pediatric patients with anti-NMDAR encephalitis in China, and whether different immunotherapies affect the long-term prognosis or not.

\section{Methods}

\section{Study design}

Pediatric patients diagnosed with anti-NMDAR encephalitis between November 2011 and December 2018 from Xiangya Hospital of Central South University, Children's Hospital of Chongqing Medical University, Peking University First Hospital, Beijing Children's Hospital, Children's Hospital of Fudan University, and Capital Institute of Pediatrics, were enrolled in our study. The inclusion criteria were: 1) meeting the diagnostic criteria for anti-NMDAR encephalitis $(8,9), 2)$ age under 18 years old, and 3) follow-up duration $>12$ months (unless the patient died from anti-NMDAR encephalitis). The exclusion criteria included: 1) lacking key clinical data, 2) being diagnosed with autoimmune diseases such as rheumatoid arthritis and lupus, or other brain disorders. Medical records including demographics, clinical characteristics, CSF examinations, brain MRI, electroencephalography (EEG), systemic screening for potential tumors, immunotherapies and relapses, as well as long-term outcome were reviewed. The follow-up was conducted through outpatient visits or telephone interviews. Relapse was defined as any 
new onset neurologic, psychiatric symptoms, or the worsening of the pre-existing symptoms after stabilization or improvement for 2 months, which could not be explained by other causes.

\section{Statistical analyses}

Statistical analyses were performed using IBM SPSS Statics 25 (SPSS Inc., Chicago, IL, USA). Treatment response and outcome analysis were assessed with mRS during follow-up. Patients were considered to have a good outcome if $m R S \leq 2$ at the last follow-up and a poor outcome if $m R S \geq 3$. Complete recovery was defined as $\mathrm{mRS}=0$. Demographic features and clinical manifestations comparing patients with good and poor outcome were analyzed with Fisher's exact test, Chi-squared test or Mann-Whitney U test when applicable. Outcomes among the treatment groups were analyzed with the Chi-squared test or Fisher's exact test. $p<0.05$ was considered significant.

\section{Results}

\section{Demographic and clinical manifestations}

A total of 386 patients were enrolled, and 224 (58.0\%) were females. The median age of symptom onset was 7.89 (range $0.5-18$ ) years. Four patients $(1.0 \%)$ were found to have an associated tumor, with two having teratoma, one having optic glioma and one having Ewing's sarcoma. All patients had mRS evaluated at symptom onset, with 324 (83.9\%) patients having $\mathrm{mRS} \geq 3$. CSF examinations showed all patients had positive anti-NMDAR antibodies, and 183 (47.4\%) patients showed other CSF abnormalities, such as mild to moderate pleocytosis and increased level of proteins. Intriguingly, anti-myelin oligodendrocyte glycoprotein (MOG) antibodies were concomitantly positive in 27 (7.0\%) patients. Abnormal signals in brain MRI were shown in 190 (49.2\%) patients. The most frequently affected locus was cerebral cortex (54.2\%), and other involved areas were basal ganglia, thalamus, hippocampus, white matter and cerebellum. Abnormal EEG activities were identified in 306 (79.3\%) patients, including epileptic discharges, diffuse slow activity, and focal slow activity.

The average duration of follow-up in our study reached to 39.2 (range 12-91.9) months. Five (1.3\%) patients were lost to follow-up. We were able to assess the final mRS in $381(98.7 \%)$ patients, and 360 $(94.5 \%)$ achieved good outcome ( $\mathrm{mRS} \leq 2)$ including 270 patients had complete recovery $(\mathrm{mRS}=0)$. By the last follow-up, $21(5.4 \%)$ patients had poor outcome (mRS $\geq 3)$ and $6(1.6 \%)$ of them died of this disease. The comparison of baseline characteristics of patients with good and poor outcome was shown in Table 1. The patients with poor outcome were more likely to be younger, have prodromal symptoms, especially fever within 3 weeks before onset, and have higher mRS at symptom onset. During the disease course, the patients with poor outcome were more likely to develop decreased level of consciousness, have abnormal brain MRI, and have unsatisfactory response to first-line immunotherapy. Pediatric intensive care unit (PICU) admission was also more frequently required in the patients with poor outcome. 
Table 1

Comparison of baseline characteristics of patients with anti-NMDAR encephalitis

\begin{tabular}{|c|c|c|c|c|}
\hline & $\begin{array}{l}\text { Good outcome } \\
(\mathrm{N}=360), \mathrm{n}(\%)\end{array}$ & $\begin{array}{l}\text { Poor outcome } \\
(\mathrm{N}=21), \mathrm{n}(\%)\end{array}$ & $x^{2}$ & $\stackrel{p}{\text { Value }}$ \\
\hline Female & $210(58.3)$ & $12(57.1)$ & 0.012 & 0.914 \\
\hline Tumor & $4(1.1)$ & $0(0)$ & - & 1.000 \\
\hline Age at symptom onset & $8.11 \pm 3.75$ & $4.97 \pm 3.98$ & $-3.420^{\mathrm{a}}$ & 0.001 \\
\hline Prodromal symptoms & $186(51.7)$ & $18(85.7)$ & 9.247 & 0.002 \\
\hline $\begin{array}{l}\text { Having fever within } 3 \text { weeks before } \\
\text { symptom onset }\end{array}$ & $129(35.8)$ & $17(81.0)$ & 17.090 & 0.000 \\
\hline Behavioral change & $305(84.7)$ & $18(85.7)$ & - & 1.000 \\
\hline Movement disorder & $281(78.1)$ & $17(81.0)$ & - & 1.000 \\
\hline Speech disorder & $263(73.1)$ & $17(81.0)$ & 0.635 & 0.425 \\
\hline Seizures & $262(72.8 \%)$ & $17(81.0)$ & 0.676 & 0.411 \\
\hline Decreased level of consciousness & $184(51.1)$ & $17(81.0)$ & 7.089 & 0.008 \\
\hline Autonomic dysfunction & $35(9.7)$ & $1(3.8)$ & - & 0.707 \\
\hline Abnormal brain MRI & $168(46.7)$ & $18(85.7)$ & 11.774 & 0.001 \\
\hline Abnormal EEG & $283(78.6)$ & $18(85.7)$ & - & 0.586 \\
\hline Abnormal CSF examinations & $168(46.7)$ & $10(47.6)$ & 0.007 & 0.932 \\
\hline mRS at symptom onset [median, (range)] & $3(1 \sim 5)$ & $4(3 \sim 5)$ & $-3.851^{\mathrm{a}}$ & 0.000 \\
\hline $\begin{array}{l}\text { mRS after first-line immunotherapy } \\
\text { [median, (range)] }\end{array}$ & $3(0-5)$ & $4(3-6)$ & $-5.182^{\mathrm{a}}$ & 0.000 \\
\hline $\begin{array}{l}\text { Days of interval between symptom onset } \\
\text { and treatments [median, (range)] }\end{array}$ & $21(1 \sim 139)$ & $23(1 \sim 63)$ & $-0.305^{a}$ & 0.760 \\
\hline Requires of PICU admission & $49(13.6)$ & $8(38.1)$ & - & 0.007 \\
\hline \multicolumn{5}{|l|}{$\therefore$ Fisher's exact test } \\
\hline a: $z$ value of non-parametric tests & & & & \\
\hline
\end{tabular}

\section{Immunotherapies}

The treatment processes of all patients were summarized in Fig. 1. The majority of patients (341, 88.3\%) used the combination of MEP and IVIG. Eighteen patients (4.7\%) additionally received plasma exchange (PLEX) on the basis of MEP. Fifteen (3.9\%) and 10 (2.6\%) patients received MEP and IVIG only, respectively. For 374 patients who used MEP treatment, 363 (97.06\%) had the dosage higher than 
$10 \mathrm{mg} / \mathrm{m}^{2} /$ day. The duration of MEP was limited within 5 days in $265(70.86 \%)$ patients. After first-line immunotherapy was completed, 375 (96.6\%) patients sequentially received high-dose oral prednisolone ( $\geq 2 \mathrm{mg} / \mathrm{kg} / \mathrm{d}$ or $60 \mathrm{mg} / \mathrm{d}$ ). Almost half of the patients $(176,46.9 \%$ ) limited the use of high-dose prednisolone to 2 weeks, and 161 (42.9\%) kept the high dosage for 2 weeks to 3 months. Treatment response of first-line immunotherapy assessed by mRS showed $175(45.3 \%)$ patients were maintained at mild conditions ( $\mathrm{mRS} \leq 2)$, whereas $211(53.9 \%)$ patients did not show satisfactory improvement (mRS $\geq 3$ ).

There were mainly 3 treatment strategies after first-line immunotherapy: second-line immunotherapy, repetitive first-line immunotherapy, and maintaining oral prednisolone.

We grouped patients according to the response to first-line immunotherapy and summarized their treatments.

In the group of patients with $m R S \geq 4(n=117), 25$ (21.4\%) received repetitive first-line immunotherapy, and 35 (29.9\%) patients received second-line immunotherapy directly. However, 15 (12.8\%) patients received both because they did not show clinical improvement after repeating first-line immunotherapy. For the patients with $\mathrm{mRS}=3(\mathrm{n}=94), 15(16.0 \%)$ patients received repetitive first-line immunotherapy, and $16(17.0 \%)$ patients used second-line immunotherapy. As for the group of patients with $m R S \leq 2(n=$ 175), the proportions of patients that received repetitive first-line immunotherapy (6.9\%) and second-line immunotherapy (3.4\%) were lower than that of other two groups. Only one patient had to go through both treatments. 41 (35.0\%), $63(67.0 \%)$ and $147(84.0 \%)$ patients maintained oral prednisolone in the groups of $m R S \geq 4, m R S=3$, and $m R S \leq 2$, respectively. Among these patients $(n=251), 170(67.7 \%)$ used longterm treatment course (>3 months), whereas 61 (24.3\%) used prednisolone for $1-3$ months, and 20 (8.0\%) tapered and stopped prednisolone within 1 month.

In terms of treatment strategies for 27 relapse patients, 4 received second-line immunotherapy, 8 repeated first-line immunotherapy and 3 used both treatments, whereas the remaining 12 patients kept oral prednisolone without any other treatments.

Eventually, a total of $80(20.7 \%)$ patients received second-line immunotherapy with 60 (75\%) using rituximab, 10 using cyclophosphamides, and 10 receiving both agents. Excluding 7 patients who used second-line immunotherapy after relapse, 57 out of 73 (78.1\%) patients started second-line immunotherapy within 4 weeks, including 35 starting within 2 weeks. Interval between first- and secondline immunotherapy longer than 4 weeks usually resulted from the use of repetitive first-line immunotherapy. For patients who repeated first-line immunotherapy $(n=52), 48.1 \%$ received the standard combination of MEP and IVIG, and $44.2 \%$ used IVIG only. The profile of repetitive first-line immunotherapy was quite different from the first time, indicating when choosing repetitive first-line immunotherapy, clinical practitioners tended to use milder regimen of monotherapy. Eighteen patients $(4.7 \%)$ received long-term immunosuppressive treatments in our series, 15 using mycophenolate mofetil, 2 using azathioprine and 1 patient receiving both, and 11 of them maintained chronic immunotherapy for more than 1 year.

\section{Outcome}


First, we analyzed whether mRS at symptom onset and after first-line immunotherapy was relevant to the outcome of patients within each treatment group (Table 2) and found no difference in complete recovery rate between patients with $\mathrm{mRS} \leq 3$ and $\mathrm{mRS} \geq 4$ at symptom onset. However, in the treatment groups of second-line immunotherapy $(0.025<p<0.05)$ and oral prednisolone $(p<0.005)$, the complete recovery rate of patients with $m R S \geq 4$ after first-line immunotherapy was lower than that of patients with $m R S \leq$ 3. Therefore, we classified patients based on their response to first-line immunotherapy and analyzed the effects of different treatment strategies on patient outcome. For patients with $m R S \geq 4$ after first-line immunotherapy, the incidence of poor outcome in oral prednisolone group was higher than that in other treatment groups (Table $3, \chi 2=4.26,0.025<p<0.05$ ). In the groups of $\mathrm{mRS}=3$ and $\leq 2$, there was no significant difference in outcome between patients from oral prednisolone group and other treatment groups (Table 3). Patients using long-term (>3 months) and short-term ( $\leq 3$ months) prednisolone showed no difference in complete recovery rate (Table 4). Moreover, patients receiving second-line, repetitive first-line immunotherapy had no difference in complete recovery rate (Table 5). It is worth noting that in the group of $\mathrm{mRS} \geq 4,15$ of 40 patients who repeated first-line immunotherapy had to receive second-line immunotherapy due to poor improvement, but this subset of patients showed no difference in complete recovery rate compared to patients from other treatment groups.

During the follow-up, 27 (7\%) patients relapsed and 25 (92.59\%) of them were from the group of maintaining oral prednisolone.

The relapse rate of patients with oral prednisolone was higher than patients of other treatment groups (Table $6, \chi^{2}=7.26, p<0.01$ ). But patients using long-term and short-term prednisolone showed no significant difference in relapse rate. 
Table 2

Relationships of mRS at symptom onset and after first-line immunotherapy and patient outcome within each treatment group.

\begin{tabular}{|c|c|c|c|c|}
\hline & $\begin{array}{l}\text { Complete } \\
\text { recovery }\end{array}$ & $\begin{array}{l}\text { Incomplete } \\
\text { recovery }\end{array}$ & $x^{2}$ & $p$ Value \\
\hline Second-line immunotherapy group & & & 1.39 & $\begin{array}{l}0.1<p< \\
0.25\end{array}$ \\
\hline \multicolumn{5}{|l|}{ mRS at symptom onset } \\
\hline $\mathrm{mRS} \leq 3$ & 10 & 4 & & \\
\hline $\mathrm{mRS} \geq 4$ & 23 & 20 & & \\
\hline mRS after first-line immunotherapy & & & 4.05 & $\begin{array}{l}0.025<p< \\
0.05\end{array}$ \\
\hline $\mathrm{mRS} \leq 3$ & 17 & 6 & & \\
\hline $\mathrm{mRS} \geq 4$ & 16 & 18 & & \\
\hline \multicolumn{5}{|l|}{$\begin{array}{l}\text { Repetitive first-line immunotherapy } \\
\text { group }\end{array}$} \\
\hline mRS at symptom onset & & & 0.21 & $\begin{array}{l}0.5<p< \\
0.75\end{array}$ \\
\hline $\mathrm{mRS} \leq 3$ & 8 & 6 & & \\
\hline $\mathrm{mRS} \geq 4$ & 21 & 21 & & \\
\hline mRS after first-line immunotherapy & & & 1.12 & $\begin{array}{l}0.25<p< \\
0.5\end{array}$ \\
\hline$m R S \leq 3$ & 17 & 12 & & \\
\hline $\mathrm{mRS} \geq 4$ & 12 & 15 & & \\
\hline Oral prednisolone group & & & 0.56 & $\begin{array}{l}0.75<p< \\
0.9\end{array}$ \\
\hline \multicolumn{5}{|l|}{ mRS at symptom onset } \\
\hline$m R S \leq 3$ & 117 & 27 & & \\
\hline$m R S \geq 4$ & 73 & 21 & & \\
\hline mRS after first-line immunotherapy & & & 12.58 & $p<0.005$ \\
\hline $\mathrm{mRS} \leq 3$ & 167 & 32 & & \\
\hline$m R S \geq 4$ & 23 & 16 & & \\
\hline
\end{tabular}


Table 3

Comparison of effects of oral prednisolone and other treatments on patient outcome

\begin{tabular}{|c|c|c|c|c|}
\hline & Good outcome & Poor outcome & $x^{2}$ & $p$ Value \\
\hline $\begin{array}{l}m R S \geq 4 \text { after first-line } \\
\text { immunotherapy }\end{array}$ & & & 4.26 & $\begin{array}{l}0.025<p< \\
0.05\end{array}$ \\
\hline Oral prednisolone & 30 & 9 & & \\
\hline Other treatments & 70 & 7 & & \\
\hline $\begin{array}{l}\mathrm{mRS}=3 \text { after first-line } \\
\text { immunotherapy }\end{array}$ & & & 0.60 & $0.25<p<0.5$ \\
\hline Oral prednisolone & 58 & 2 & & \\
\hline \multirow[t]{2}{*}{ Other treatments } & 28 & 3 & & \\
\hline & $\begin{array}{l}\text { Complete } \\
\text { recovery }\end{array}$ & $\begin{array}{l}\text { Incomplete } \\
\text { recovery }\end{array}$ & & \\
\hline $\begin{array}{l}\text { mRS } \leq 2 \text { after first-line } \\
\text { immunotherapy }\end{array}$ & & & 0.58 & $0.25<p<0.5$ \\
\hline Oral prednisolone & 115 & 24 & & \\
\hline Other treatments & 27 & 8 & & \\
\hline
\end{tabular}

Table 4

Comparison of effects of long-term and short-term prednisolone on patient outcome

\begin{tabular}{|lllll|}
\hline & $\begin{array}{l}\text { Complete } \\
\text { recovery }\end{array}$ & $\begin{array}{l}\text { Incomplete } \\
\text { recovery }\end{array}$ & $\chi 2$ & $p$ Value \\
\hline $\begin{array}{l}\text { mRS } \geq 3 \text { after first-line } \\
\text { immunotherapy }\end{array}$ & & & 0.31 & $\begin{array}{l}0.5<p< \\
0.75\end{array}$ \\
$\leq 3$ months & 33 & 9 & & \\
$>3$ months & 42 & 15 & 0.013 & $p>0.9$ \\
$\begin{array}{l}\text { mRS } \leq 2 \text { after first-line } \\
\text { immunotherapy }\end{array}$ & & & & \\
\hline$\leq 3$ months & 30 & 6 & & \\
\hline$>3$ months & 85 & 18 & & \\
\hline
\end{tabular}


Table 5

Comparison of effects of second-line and repetitive first-line immunotherapy on patient outcome

\begin{tabular}{|c|c|c|c|c|}
\hline & $\begin{array}{l}\text { Complete } \\
\text { recovery }\end{array}$ & $\begin{array}{l}\text { Incomplete } \\
\text { recovery }\end{array}$ & $x^{2}$ & $p$ Value \\
\hline $\begin{array}{l}\mathrm{mRS} \geq 4 \text { after first-line } \\
\text { immunotherapy }\end{array}$ & & & 0.04 & $p>0.975$ \\
\hline Second-line immunotherapy & 16 & 18 & & \\
\hline Repetitive first-line immunotherapy & 12 & 15 & & \\
\hline Both & 7 & 8 & & \\
\hline $\begin{array}{l}\text { mRS }=3 \text { after first-line } \\
\text { immunotherapy }\end{array}$ & & & 0.81 & $\begin{array}{l}0.25<p< \\
0.5\end{array}$ \\
\hline Second-line immunotherapy & 9 & 4 & & \\
\hline Repetitive first-line immunotherapy & 9 & 8 & & \\
\hline
\end{tabular}

\section{Table 6}

Comparison of effects of different immunotherapy strategies on relapse rate of patients with anti-NMDAR encephalitis

\begin{tabular}{|lllll|}
\hline & Relapse & Non-relapse & $\chi 2$ & $p$ Value \\
\hline Immunotherapies & & & 7.26 & $p<0.01$ \\
\hline Oral prednisolone & 25 & 226 & & \\
\hline Other treatment groups & 2 & 133 & & \\
\hline Treatment course of oral prednisolone & & & - & $p=0.942$ \\
\hline$\leq 1$ month & 2 & 18 & & \\
\hline$>1$ month, $\leq 3$ months & 5 & 56 & & \\
\hline$>3$ months & 18 & 152 & & \\
\hline
\end{tabular}

\section{Discussion}

Pediatric patients accounted for approximately $40 \%$ of reported cases of anti-NMDAR encephalitis $(3,10)$. To our knowledge, multicenter studies of immunotherapies of pediatric anti-NDMAR encephalitis across multiple areas in China are rare. At symptom onset, the percentage of patients with mRS $\geq 3$ was $83.9 \%$, which was decreased to $54.7 \%$ after first-line immunotherapy. Second-line immunotherapy is recommended to administrate to patients who do not have good response to first-line immunotherapy. However, using mRS to assess treatment response can only reflect the conditions of patients at specific time points but fails to show the tendency of the disease. In reality, prediction to the progression of disease from practitioners' perspective, as well as many other factors, such as costs, hospitalization 
requirements and concerns about side effects, have to be taken into consideration when applying secondline immunotherapy.

In $m R S \geq 4$ group, patients taking oral prednisolone showed unfavorable outcome compared to patients receive other treatments.

Therefore, for patients with $\mathrm{mRS} \geq 4$ after first-line immunotherapy, aggressive treatments are recommended and use of oral prednisolone without any other treatments should be avoided. Patients using second-line and repetitive first-line immunotherapy did not show significant difference in complete recovery rate and $37.5 \%$ of patients who repeated first-line immunotherapy still needed second-line immunotherapy due to unsatisfactory improvement, but this subset of patients showed no difference in complete recovery rate compared to patients from other treatment groups, suggesting repetitive first-line immunotherapy can be considered when second-line immunotherapy is not applicable due to severe adverse effects or high costs, and delaying second-line immunotherapy in patients with repetitive first-line immunotherapy did not affect outcome.

We found the relapse rate of oral prednisolone group was higher than that of other treatment groups, indicating using second-line or repetitive first-line immunotherapy was beneficial to reduce relapses. More than half of patients who kept on oral prednisolone in our series used long-term regimen ( $>3$ months). However, long-term use of corticosteroid can cause multiple adverse effects, such as cushingoid features (redistribution of body fat with truncal obesity, buffalo hump, and moon face), osteoporosis, infections, growth impairments and so on. We found no difference in complete recovery rate and relapse rate between patients using short-term ( $\leq 3$ months) and long-term (>3 months) regimens. Therefore, shortening the duration of prednisolone use is worth considering after fully evaluating patients' conditions.

However, there are limitations to our study. First, selection bias may exist because all hospitals involved in our study are major tertiary hospitals that usually accept more patients with critical conditions than hospitals in rural area. Second, evaluation of outcomes was conducted at different time points across patients. Since we are aware that recovery from this disease can be slow and take 18 months or longer. Many patients can recover further as follow-up duration extending. Third, the outcome evaluated via mRS is dichotomous, focusing on the locomotor performance. However, multiple studies described pediatric patients could show some neuropsychological sequela, such as cognitive and social functioning deficits, even up until adolescence, causing learning issues (11-13). Thus, prospective longitudinal studies will be required to have better control of these confounding factors to pursue better interventions for this disease.

\section{Conclusions}

In conclusion, for patients with $\mathrm{mRS} \geq 4$ after first-line immunotherapy, we recommend more aggressive treatments like second-line immunotherapy. When second-line immunotherapy is not applicable due to severe adverse effects or other reasons, repetitive first-line immunotherapy can be considered as an 
option. Both second-line and repetitive first-line immunotherapy are beneficial to reduce relapse rate. The treatment course of sequential oral prednisolone, as a routine maintenance treatment, can be shortened after fully evaluate patients' conditions.

\section{Abbreviations}

NMDAR = N-methyl-D-aspartate receptor; MEP = methylprednisolone pulses; IVIG = intravenous immunoglobulins; $\mathrm{PICU}=$ pediatric intensive care unit

\section{Declarations}

\section{Ethics approval and consent to participate}

This study has been reviewed and approved by the Institutional Ethics Committee of Xiangya Hospital of Central South University. The Chinese Medical Association waived the requirement to obtain patient informed consent to access medical records.

\section{Consent for publication}

Not applicable.

\section{Availability of data and materials}

The datasets used and/or analysed during the current study are available from the corresponding author on reasonable request.

\section{Competing interests}

The authors declare that they have no competing interests.

\section{Funding}

This study is funded by National Natural Science Foundation of China (82071462).

\section{Authors' contributions}

analyzed the data and revised the manuscript for intellectual content.

\section{Acknowledgements}

Not applicable.

\section{References}


1. Dalmau J, Tüzün E, Wu HY, et al. Paraneoplastic anti-N-methyl-D-aspartate receptor encephalitis associated with ovarian teratoma. Annals of neurology. 2007;61(1):25-36.

2. Vitaliani R, Mason W, Ances B, et al. Paraneoplastic encephalitis, psychiatric symptoms, and hypoventilation in ovarian teratoma. Annals of neurology. 2005;58(4):594-604.

3. Titulaer MJ, McCracken L, Gabilondo I, et al. Treatment and prognostic factors for long-term outcome in patients with anti-NMDA receptor encephalitis: an observational cohort study. The Lancet Neurology. 2013;12(2):157-65.

4. Zhang L, Wu MQ, Hao ZL, et al. Clinical characteristics, treatments, and outcomes of patients with anti-N-methyl-d-aspartate receptor encephalitis: A systematic review of reported cases. Epilepsy \& behavior : E\&B. 2017;68:57-65.

5. Bartolini L, Muscal E. Differences in treatment of anti-NMDA receptor encephalitis: results of a worldwide survey. Journal of neurology. 2017;264(4):647-53.

6. Kahn I, Helman G, Vanderver A, Wells E. Anti- N-Methyl-d-Aspartate (NMDA) Receptor Encephalitis. Journal of child neurology. 2017;32(2):243-5.

7. Cao XS, Kessi M, Ji TY, et al. A survey on pediatric anti-N-methyl-D-aspartate-receptor encephalitis treatment strategies in China. Chinese medical journal. 2020.

8. Cellucci T, Van Mater H, Graus F, et al. Clinical approach to the diagnosis of autoimmune encephalitis in the pediatric patient. Neurology(R) neuroimmunology \& neuroinflammation. 2020;7(2).

9. Graus F, Titulaer MJ, Balu R, et al. A clinical approach to diagnosis of autoimmune encephalitis. The Lancet Neurology. 2016;15(4):391-404.

10. Florance NR, Davis RL, Lam C, et al. Anti-N-methyl-D-aspartate receptor (NMDAR) encephalitis in children and adolescents. Annals of neurology. 2009;66(1):11-8.

11. de Bruijn M, Aarsen FK, van Oosterhout MP, et al. Long-term neuropsychological outcome following pediatric anti-NMDAR encephalitis. Neurology. 2018;90(22):e1997-e2005.

12. Iadisernia E, Battaglia FM, Vanadia E, et al. Anti-N-methyl-D-aspartate-receptor encephalitis: cognitive profile in two children. European journal of paediatric neurology : EJPN : official journal of the European Paediatric Neurology Society. 2012;16(1):79-82.

13. Matricardi S, Patrini M, Freri E, et al. Cognitive and neuropsychological evolution in children with antiNMDAR encephalitis. Journal of neurology. 2016;263(4):765-71.

\section{Figures}




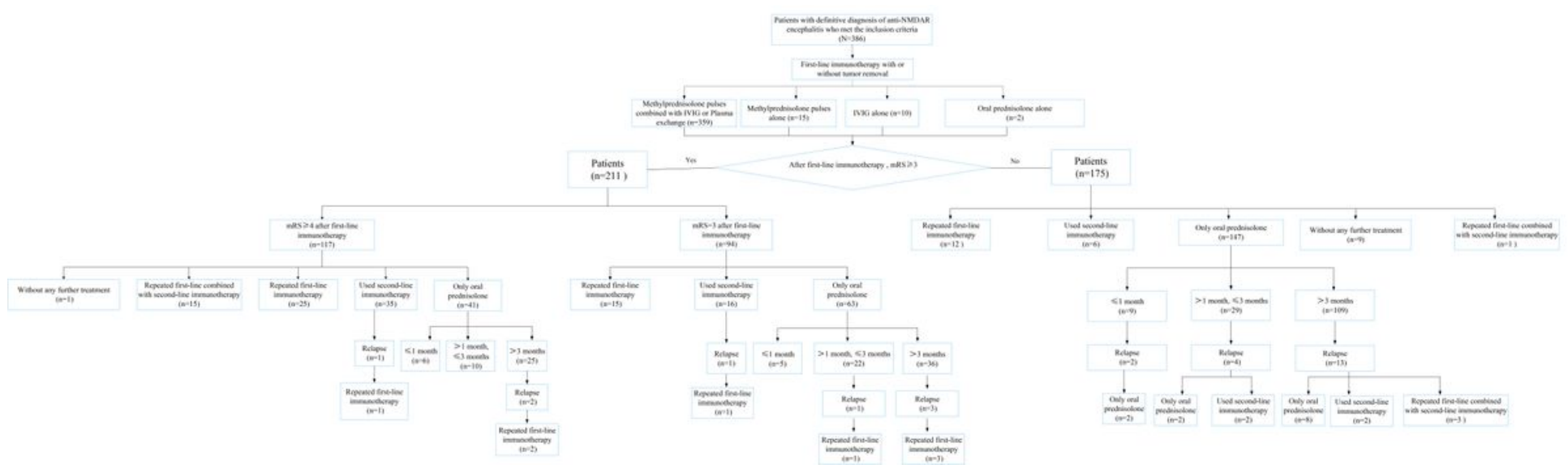

Figure 1

Treatment strategies for pediatric patients with anti-NMDAR encephalitis 